Boise State University

ScholarWorks

Mathematics Faculty Publications and

Presentations

Department of Mathematics

6-3-2016

\title{
Maximum Waring Ranks of Monomials and Sums of Coprime Monomials
}

\author{
Erik Holmes \\ University of Hawaii at Manoa \\ Paul Plummer \\ University of Oklahoma \\ Jeremy Siegert \\ George Washington University \\ Zach Teitler \\ Boise State University
}

This is an Accepted Manuscript of an Article published in Communications in Algebra (2016) available online at doi: 10.1080/00927872.2015.1087534 
This is an author-produced, peer-reviewed version of this article. The final, definitive version of this document can be found online at Communications in Algebra, published by Taylor \& Francis. Copyright restrictions may apply. doi: 10.1080/00927872.2015.1087534

\title{
MAXIMUM WARING RANKS OF MONOMIALS AND SUMS OF COPRIME MONOMIALS
}

\author{
ERIK HOLMES, PAUL PLUMMER, JEREMY SIEGERT, AND ZACH TEITLER
}

\begin{abstract}
We show that monomials and sums of pairwise coprime monomials in four or more variables have Waring rank less than the generic rank, with a short list of exceptions. We asymptotically compare their ranks with the generic rank.
\end{abstract}

\section{INTRODUCTION}

Let $F\left(x_{1}, \ldots, x_{n}\right)$ be homogeneous polynomial of degree $d$, a $d$-form, in the variables $x_{1}, \ldots, x_{n}$ with complex coefficients. The Waring rank of $F$, denoted $\mathrm{r}(F)$, is the least number of terms needed to write $F$ as a linear combination of $d$ th powers of linear polynomials, $F=c_{1} \ell_{1}^{d}+\cdots+c_{r} \ell_{r}^{d}$. For example, $F(x, y)=x y$ can be written as

$$
x y=\frac{1}{4}(x+y)^{2}-\frac{1}{4}(x-y)^{2}
$$

which shows $\mathrm{r}(x y) \leq 2$. On the other hand we must have $\mathrm{r}(x y)>1$ because if $x y=c_{1} \ell_{1}^{2}$ then $x y$ would be a perfect square, which it is not. Therefore $\mathrm{r}(x y)=2$.

Waring ranks of homogeneous forms have been studied since the 19th century by Sylvester and others. For modern introductions see for example [14], [17], [21]. For numerous applications in engineering, sciences, and other areas of mathematics, see for example [11], [17].

We write $\mathrm{r}_{\text {gen }}(n, d)$ for the rank of a general $d$-form in $n$ variables. This value, called the generic rank, is well-known by the Alexander-Hirschowitz theorem, see Section 2.

In contrast it is difficult to determine the maximum Waring rank occuring for $d$-forms in $n$ variables. Clearly the maximum value of rank is at least the generic value. In [5] it is shown that the maximum value of rank is at most twice the generic value, so the candidates for the maximum rank are the integers in the interval $\left[\mathrm{r}_{\text {gen }}(n, d), 2 \mathrm{r}_{\text {gen }}(n, d)\right]$. We can narrow this range either by finding new upper bounds or by finding forms with greater than generic rank.

Another reason to look for forms of high rank is that such examples may have interesting applications in the theory of computational complexity. See for example [2], [20] for discussions of complexity-theoretic conclusions from tensors of high rank. See [18] for a more general introduction to connections between geometry and complexity.

We examine some candidate forms - monomials and sums of pairwise coprime monomialsand find that in most cases they fail to have greater than generic rank.

It turns out that very few examples are known of forms with greater than generic rank, with $n \geq 3$ variables. (Plenty are known for $n=2$.) In fact, it seems that until recently only

Date: August 20, 2015.

2010 Mathematics Subject Classification. 13F20.

Key words and phrases. Waring rank, Waring problem for homogeneous polynomials, maximum Waring rank, upper bounds for Waring rank. 
This is an author-produced, peer-reviewed version of this article. The final, definitive version of this document can be found online at Communications in Algebra, published by Taylor \& Francis. Copyright restrictions may apply. doi: 10.1080/00927872.2015.1087534

finitely many such examples were known: just some cubics and quartics $(d=3,4)$ in $n=3$ or 4 variables, see Section 2. (See also [7].)

Recently, however, Carlini, Catalisano, and Geramita pointed out an infinite family of forms with greater than generic rank. Specifically, they showed in [9] (see also [6]) that for a monomial $M=x_{1}^{a_{1}} \cdots x_{n}^{a_{n}}$ with $0<a_{1} \leq \cdots \leq a_{n}$, the Waring rank of $M$ is

$$
\mathrm{r}(M)=\left(a_{2}+1\right) \cdots\left(a_{n}+1\right) \text {. }
$$

We denote by $\overline{\mathrm{r}}_{\text {mon }}(n, d)$ the maximum rank of a monomial in $n$ variables of degree $d$. CarliniCatalisano-Geramita observed that for $n=3$ and $d \geq 5$ there are monomials of greater than generic rank, $\overline{\mathrm{r}}_{\text {mon }}(3, d)>\mathrm{r}_{\text {gen }}(3, d)$ for $d \geq 5$. And they observed also that for $n \geq$ 4 , $\lim _{d \rightarrow \infty} \overline{\mathrm{r}}_{\text {mon }}(n, d) / \mathrm{r}_{\text {gen }}(n, d)<1$, so for each $n \geq 4$ there are (at most) finitely many monomials with greater than generic rank.

Our first main result is that in fact, in four or more variables there are absolutely no monomials with higher than generic rank.

Theorem 1. Let $M$ be a monomial in $n \geq 4$ variables and let $d=\operatorname{deg} M>1$. Then $\mathrm{r}(M)<\mathrm{r}_{\text {gen }}(n, d)$. That is, $\overline{\mathrm{r}}_{\text {mon }}(n, d)<\mathrm{r}_{\text {gen }}(n, d)$ whenever $n \geq 4$ and $d>1$.

This is proved in Section 3.

Carlini-Catalisano-Geramita showed also in [9] that for a sum of coprime monomials $F=M_{1}+\cdots+M_{k}$, where the terms are monomials in independent sets of variables,

$$
\mathrm{r}\left(M_{1}+\cdots+M_{t}\right)=\mathrm{r}\left(M_{1}\right)+\cdots+\mathrm{r}\left(M_{t}\right) .
$$

(It is conjectured that such an equality holds for any sum of polynomials in independent sets of variables, see for example [8].)

Example 2. The form $F=x_{1} x_{2}^{2}+x_{3} x_{4}^{2}$, with $n=4, d=3$, has higher than generic rank:

$$
\mathrm{r}\left(x_{1} x_{2}^{2}+x_{3} x_{4}^{2}\right)=\mathrm{r}\left(x_{1} x_{2}^{2}\right)+\mathrm{r}\left(x_{3} x_{4}^{2}\right)=6>\mathrm{r}_{\text {gen }}(4,3)=5 .
$$

The forms $x_{1} x_{2} x_{3}+x_{4}^{3}$ and $x_{1} x_{2}^{2}+x_{3}^{3}+x_{4}^{3}$ each have rank 5 , equal to the generic rank.

In fact these are the only sums of coprime monomials with greater than or equal to generic rank (up to reordering the variables). We denote by $\overline{\mathrm{r}}_{\Sigma \text {-mon }}(n, d)$ the maximum rank of a sum of coprime monomials in $n$ (total) variables of degree $d$.

Theorem 3. Every sum of pairwise coprime monomials in $n \geq 4$ variables, of degree $d \geq 3$, has rank strictly less than the generic rank, except for the three forms listed in Example 2. That is, $\overline{\mathrm{r}}_{\Sigma \text {-mon }}(n, d)<\mathrm{r}_{\text {gen }}(n, d)$ whenever $n \geq 4$ and $d \geq 3$, except for $(n, d)=(4,3)$.

This is proved in Section 4.

Finally we asymptotically compare the maximum ranks of sums of pairwise coprime monomials with the generic rank.

\section{BACKGROUND}

By the Alexander-Hirschowitz theorem [1] the generic rank is given by

$$
\mathrm{r}_{\mathrm{gen}}(n, d)=\left\lceil\frac{1}{n}\left(\begin{array}{c}
d+n-1 \\
n-1
\end{array}\right)\right\rceil,
$$

except if $(n, d)=(n, 2),(3,4),(4,4),(5,3),(5,4)$. In the exceptional cases $\mathrm{r}_{\text {gen }}(n, 2)=n$ $($ instead of $\lceil(n+1) / 2\rceil), \mathrm{r}_{\text {gen }}(3,4)=6($ instead of 5$), \mathrm{r}_{\text {gen }}(4,4)=10($ instead of 9$), \mathrm{r}_{\text {gen }}(5,3)=$ 8 (instead of 7$)$, and $r_{\text {gen }}(5,4)=15$ (instead of 14$)$. 
Having said that, we will only occasionally need information about the exceptional cases (and we will in fact never need to know that these are the only exceptional cases, which is the significant part of the Alexander-Hirschowitz theorem). At most points we will just need that $\mathrm{r}_{\text {gen }}(n, d) \geq \frac{1}{n}\left(\begin{array}{c}d+n-1 \\ n-1\end{array}\right)$ for all $n, d \geq 2$, which follows from a standard dimension count.

For some recent progress on upper bounds for Waring rank, see [4], [15], [3], and [5]. But there are only a few cases in which the actual maximum rank, or even explicit forms of greater than generic rank, are known. Binary $(n=2)$ forms of degree $d$ have rank at most $d$, with $\mathrm{r}\left(x y^{d-1}\right)=d$. For quadratic forms $(d=2)$ the maximum rank is $n$. For $(n, d)=(3,3)$, $\mathrm{r}_{\text {gen }}(3,3)=4$ while the maximum rank is 5 [22, §96], [11], [16], [19, §8]. For $(n, d)=(3,4)$, $\mathrm{r}_{\text {gen }}(3,4)=6$ while the maximum rank is 7 [16], [12]. For $(n, d)=(4,3), \mathrm{r}_{\text {gen }}(4,3)=5$ while the maximum rank is again 7 [22, §97]. Finally, very recently it has been determined that for $(n, d)=(4,4)$ the maximum rank is 10 [13], [7] (the latter gives an explicit form of rank $10)$.

For $(n, d)=(3,3)$, each one of $[22, \S 96],[11],[16],[19, \S 8]$ shows that, up to a linear change of coordinates, the unique form of greater than generic rank is $z\left(x^{2}+y z\right)$, the union of a smooth conic and a tangent line. For $(n, d)=(3,4), z^{2}\left(x^{2}+y z\right)$ is the unique form (again up to a linear change of coordinates) of greater than generic rank, see [16, Proposition 3.1]. For $(n, d)=(4,3), w\left(x^{2}+y^{2}+z w\right)$, the union of a smooth quadric and a tangent plane, has rank 7, greater than the generic rank [22, §97]. (In this case there are other forms with greater than generic rank, see $[22, \S 97]$.) For more on hypersurfaces consisting of a quadric plus a hyperplane see [10].

\section{RANKS OF MONOMIALS IN FOUR OR MORE VARIABLES}

Proof of Theorem 1. Let $M$ be a monomial in $n \geq 4$ variables and let $d=\operatorname{deg} M>1$. We do not assume that $M$ actually involves every variable.

Say $k \leq n$ of the variables appear in $M=x_{1}^{a_{1}} \cdots x_{k}^{a_{k}}, 0<a_{1} \leq \cdots \leq a_{k}$. Write $M=x_{1}^{a_{1}} \cdots x_{n}^{a_{n}}, a_{k+1}=\cdots=a_{n}=0$. By the arithmetic-geometric mean inequality,

$$
\begin{aligned}
& \mathrm{r}(M)=\left(a_{2}+1\right) \cdots\left(a_{n}+1\right) \leq\left(\frac{a_{2}+\cdots+a_{n}+n-1}{n-1}\right)^{n-1} \\
& \quad=\left(\frac{d+n-1-a_{1}}{n-1}\right)^{n-1} \leq\left(\frac{d+n-2}{n-1}\right)^{n-1} .
\end{aligned}
$$

We finish by the following lemma.

Lemma 4. For $n \geq 4$ and $d \geq 2$,

$$
\left(\frac{d+n-2}{n-1}\right)^{n-1}<\frac{1}{n}\left(\begin{array}{c}
d+n-1 \\
n-1
\end{array}\right) .
$$

Proof. We will show the equivalent equation

$$
\left(\frac{d+n-2}{d+n-1}\right) \cdots\left(\frac{d+n-2}{d+1}\right)<\frac{(n-1)^{n-1}}{n !}=\left(\frac{n-1}{n}\right) \cdots\left(\frac{n-1}{2}\right) .
$$

First, it is easy to check that

$$
\left(\frac{d+n-2}{d+n-1}\right)\left(\frac{d+n-2}{d+n-3}\right)<\left(\frac{n-1}{n}\right)\left(\frac{n-1}{n-2}\right)
$$


This takes care of the first three factors on each side in (2). (Here we use the hypothesis $n \geq 4$; otherwise $\frac{d+n-2}{d+n-3}$ and $\frac{n-1}{n-2}$ are absent.) For the remaining factors,

$$
\frac{(d-1)+(n-1)}{(d-1)+a}<\frac{n-1}{a}
$$

for $2 \leq a<n-1$. This proves (2) and completes the proof.

\section{SUMS OF PAIRWISE COPRIME MONOMIALS}

Lemma 5. If $d \geq 4$ and $d \geq n \geq 2$, then $\frac{1}{n} \overline{\mathrm{r}}_{\text {mon }}(n, d) \geq \frac{1}{n-1} \overline{\mathrm{r}}_{\text {mon }}(n-1, d)$.

Proof. First, if $n=2, \overline{\mathrm{r}}_{\text {mon }}(2, d)=d$ while $\overline{\mathrm{r}}_{\text {mon }}(1, d)=1$, so the claim is true. Second, suppose $d>n>2$. Let $M$ be a monomial in $n-1$ variables of degree $d$ with rank $\overline{\mathrm{r}}_{\text {mon }}(n-1, d)$. Up to reordering the variables, $M=x_{1}^{1} x_{2}^{a_{2}} \cdots x_{n-1}^{a_{n-1}}$ with $a_{2} \leq \cdots \leq a_{n-1} \leq$ $a_{2}+1$. We have

$$
a_{n-1}=\left\lceil\frac{d-1}{n-2}\right\rceil>1
$$

Let $M^{\prime}=x_{1}^{1} x_{2}^{a_{2}} \cdots x_{n-1}^{a_{n-1}-1} x_{n}^{1}$, so $M^{\prime}$ still has degree $d$, and

$$
\begin{aligned}
\overline{\mathrm{r}}_{\text {mon }}(n, d) & \geq \mathrm{r}\left(M^{\prime}\right) \\
& =\mathrm{r}(M) \frac{2 a_{n-1}}{a_{n-1}+1} \\
& =\overline{\mathrm{r}}_{\text {mon }}(n-1, d) \frac{2\left\lceil\frac{d-1}{n-2}\right\rceil}{\left\lceil\frac{d-1}{n-2}\right\rceil+1} \\
& \geq \overline{\mathrm{r}}_{\text {mon }}(n-1, d) \frac{2(d-1)}{d+n-3} \\
& \geq \overline{\mathrm{r}}_{\text {mon }}(n-1, d) \frac{n}{n-1},
\end{aligned}
$$

where the first equality comes from the Carlini-Catalisano-Geramita expression for Waring rank of monomials and the remaining steps are straightforward algebraic manipulations.

Finally, if $d=n \geq 4$ then $\overline{\mathrm{r}}_{\text {mon }}(n, d)=2^{d-1}$ and $\overline{\mathrm{r}}_{\text {mon }}(n-1, d)=3 \cdot 2^{d-3}=\frac{3}{4} \overline{\mathrm{r}}_{\text {mon }}(n, d)$. Since $n \geq 4, \frac{n-1}{n} \geq \frac{3}{4}$, so $\frac{1}{n} \overline{\mathrm{r}}_{\text {mon }}(n, d) \geq \frac{1}{n-1} \overline{\mathrm{r}}_{\text {mon }}(n-1, d)$.

Proof of Theorem 3. First suppose $d \geq n \geq 4$. Let $F=M_{1}+\cdots+M_{t}$ be a sum of pairwise coprime monomials of degree $d$, where $M_{i}$ involves exactly $n_{i}$ variables, $n=\sum n_{i}$, $n_{1} \geq \cdots \geq n_{t} \geq 1$. For each $i$,

$$
\frac{\mathrm{r}\left(M_{i}\right)}{n_{i}} \leq \frac{\overline{\mathrm{r}}_{\text {mon }}\left(n_{i}, d\right)}{n_{i}} \leq \frac{\overline{\mathrm{r}}_{\text {mon }}(n, d)}{n}
$$

by Lemma 5. We use the elementary inequality that if $a_{i}, b_{i}>0$ and $\frac{a_{i}}{b_{i}} \leq x$ for all $i$, then $\left(\sum a_{i}\right) /\left(\sum b_{i}\right) \leq x$. Thus

$$
\frac{\mathrm{r}(F)}{n}=\frac{\sum \mathrm{r}\left(M_{i}\right)}{\sum n_{i}} \leq \frac{\overline{\mathrm{r}}_{\mathrm{mon}}(n, d)}{n}
$$

hence $\mathrm{r}(F) \leq \overline{\mathrm{r}}_{\text {mon }}(n, d)$. That is, for $d \geq n \geq 4, \overline{\mathrm{r}}_{\Sigma \text {-mon }}(n, d)=\overline{\mathrm{r}}_{\text {mon }}(n, d)<\mathrm{r}_{\text {gen }}(n, d)$.

Second we deal with the case $n>d \geq 4$. We will use that

$$
\frac{1}{n^{2}}\left(\begin{array}{c}
d+n-1 \\
n-1
\end{array}\right)>\frac{2^{d-1}}{d}
$$


We prove this by induction on $n$ starting from $n=d \geq 4$, where we have $\frac{1}{d^{2}}\left(\begin{array}{c}2 d-1 \\ d-1\end{array}\right)>\frac{2^{d-1}}{d}$ by Lemma 4. And for $n \geq d \geq 4$ we have

$$
\begin{aligned}
\frac{1}{n^{2}}\left(\begin{array}{c}
d+n-1 \\
n-1
\end{array}\right) & =\frac{1}{n^{2}} \cdot \frac{n}{n+d}\left(\begin{array}{c}
d+n \\
n
\end{array}\right) \\
& =\frac{(n+1)^{2}}{n(n+d)} \cdot \frac{1}{(n+1)^{2}}\left(\begin{array}{c}
d+n \\
n
\end{array}\right) \\
& <\frac{1}{(n+1)^{2}}\left(\begin{array}{c}
d+n \\
n
\end{array}\right)
\end{aligned}
$$

since $d>2$. This completes the proof of (3).

Now let $F=M_{1}+\cdots+M_{t}$ be a sum of pairwise coprime monomials of degree $d$, where $M_{i}$ involves exactly $n_{i}$ variables, $n=\sum n_{i}, n_{1} \geq \cdots \geq n_{t} \geq 1$. Note each $n_{i} \leq d$. We have

$$
\frac{\mathrm{r}\left(M_{i}\right)}{n_{i}} \leq \frac{\overline{\mathrm{r}}_{\mathrm{mon}}(d, d)}{d}=\frac{2^{d-1}}{d} \text {. }
$$

Therefore

$$
\frac{\mathrm{r}(F)}{n}=\frac{\sum \mathrm{r}\left(M_{i}\right)}{\sum n_{i}} \leq \frac{2^{d-1}}{d}<\frac{1}{n^{2}}\left(\begin{array}{c}
d+n-1 \\
n-1
\end{array}\right)
$$

by (3), which gives us

$$
\mathrm{r}(F)<\frac{1}{n}\left(\begin{array}{c}
d+n-1 \\
n-1
\end{array}\right) \leq \mathrm{r}_{\text {gen }}(n, d)
$$

as desired. This completes the case $n>d \geq 4$.

Third we take care of the case $d=3, n \geq 5$. Let $F$ be a sum of pairwise coprime monomials of degree 3 with rank $\overline{\mathrm{r}}_{\Sigma \text {-mon }}(n, 3)$. The only monomials that can appear are of the form $x^{3}$, $x y^{2}, x y z$, with ranks $1,3,4$ respectively. We can replace each occurence in $F$ of $x y z$ with $x y^{2}+z^{3}$ without changing the rank or number of variables. So we can assume every term in $F$ is of the form $x^{3}$ or $x y^{2}$. This shows that if $n$ is even, $\overline{\mathrm{r}}_{\Sigma \text {-mon }}(n, 3)=3 n / 2$, and if $n$ is odd, $\overline{\mathrm{r}}_{\Sigma \text {-mon }}(n, 3)=(3 n-1) / 2$. On the other hand,

$$
\mathrm{r}_{\mathrm{gen}}(n, 3) \geq \frac{1}{n}\left(\begin{array}{c}
n+2 \\
3
\end{array}\right)=\frac{3 n}{2}+\frac{n(n-6)+2}{6} .
$$

When $n \geq 6, n(n-6)+2 \geq 2$, which shows $\mathrm{r}_{\text {gen }}(n, 3)>\frac{3 n}{2} \geq \overline{\mathrm{r}}_{\Sigma \text {-mon }}(n, 3)$. When $n=5$, $\mathrm{r}_{\text {gen }}(5,3)=8$ (by the Alexander-Hirschowitz theorem) while $\overline{\mathrm{r}}_{\Sigma \text {-mon }}(5,3)=7$.

Fourth and finally, we consider the case $(n, d)=(4,3)$. Up to reordering terms and variables, the sums of pairwise coprime monomials that use all the variables are the following: $x_{1}^{3}+x_{2}^{3}+x_{3}^{3}+x_{4}^{3}, x_{1} x_{2}^{2}+x_{3}^{3}+x_{4}^{3}, x_{1} x_{2} x_{3}+x_{4}^{3}$, and $x_{1} x_{2}^{2}+x_{3} x_{4}^{2}$; and these have rank 4 , 5,5 , and 6 , respectively. Since $\mathrm{r}_{\text {gen }}(4,3)=5$, this shows that the exceptions listed in the statement of the theorem are the only ones.

Remark 6 . We have seen that if $d \geq n$ then $\overline{\mathrm{r}}_{\Sigma \text {-mon }}(n, d)$ is attained by a monomial. What if $n>d$ ? The greatest rank monomial of degree $d$ is a product of $d$ variables. So a "greedy" way to construct a high-rank sum of pairwise coprime monomials is to add up products of $d$ variables, with any remaining variables placed into one more monomial. But this does not necessarily maximize Waring rank, as we have seen for $(n, d)=(4,3)$ : the greedy choice $x_{1} x_{2} x_{3}+x_{4}^{3}$ has rank 5 , while the non-greedy choice $x_{1} x_{2}^{2}+x_{3} x_{4}^{2}$ has rank 6 . Similarly, for $(n, d)=(5,4)$, the greedy choice $x_{1} x_{2} x_{3} x_{4}+x_{5}^{4}$ has rank 9 , while the non-greedy choice 
$x_{1} x_{2} x_{3}^{2}+x_{4} x_{5}^{3}$ has rank 10 ; and for $(n, d)=(6,5)$, the greedy choice $x_{1} \cdots x_{5}+x_{6}^{5}$ has rank 17 , while the non-greedy choice $x_{1} x_{2}^{2} x_{3}^{2}+x_{4} x_{5}^{2} x_{6}^{2}$ has rank 18 .

Remark 7. It was noted in [9] that, for $n$ fixed and $d$ going to infinity, $\overline{\mathrm{r}}_{\text {mon }}(n, d)$ is asymptotically $d^{n-1} /(n-1)^{n-1}$, while $\mathrm{r}_{\text {gen }}(n, d)$ is asymptotically $d^{n-1} / n !$. So $\overline{\mathrm{r}}_{\text {mon }}(3, d) / \mathrm{r}_{\text {gen }}(3, d) \rightarrow$ $3 / 2$, while for $n>3$,

$$
\frac{\overline{\mathrm{r}}_{\text {mon }}(n, d)}{\mathrm{r}_{\text {gen }}(n, d)} \rightarrow \frac{n !}{(n-1)^{n-1}}<1 \quad \text { as } d \rightarrow \infty .
$$

Similarly, if $n \geq 4$ is fixed and $d \rightarrow \infty$, then, for $d \geq n, \overline{\mathrm{r}}_{\Sigma \text {-mon }}(n, d)=\overline{\mathrm{r}}_{\text {mon }}(n, d)$, and once again,

$$
\frac{\overline{\mathrm{r}}_{\Sigma-\mathrm{mon}}(n, d)}{\mathrm{r}_{\text {gen }}(n, d)} \rightarrow \frac{n !}{(n-1)^{n-1}}<1 \quad \text { as } d \rightarrow \infty .
$$

Finally, fix $d$ to find the limit of the ratio as $n \rightarrow \infty$. In the proof of Theorem 3 we found that, for a fixed $d \geq 3, \overline{\mathrm{r}}_{\Sigma \text {-mon }}(n, d)$ is bounded by a linear function for large enough $n: \overline{\mathrm{r}}_{\Sigma \text {-mon }}(n, d) \leq \frac{3 n}{2}$ when $d=3$ and $\overline{\mathrm{r}}_{\Sigma \text {-mon }}(n, d) \leq \frac{n 2^{d-1}}{d}$ when $n>d \geq 4$, by (4). However for $d \geq 3, \mathrm{r}_{\text {gen }}(n, d)=O\left(n^{d-1}\right)$ grows faster than a linear function of $n$, so

$$
\frac{\overline{\mathrm{r}}_{\Sigma-\mathrm{mon}}(n, d)}{\mathrm{r}_{\text {gen }}(n, d)} \rightarrow 0 \quad \text { as } n \rightarrow \infty \text {. }
$$

\section{REFERENCES}

1. J. Alexander and A. Hirschowitz, Polynomial interpolation in several variables, J. Algebraic Geom. 4 (1995), no. 2, 201-222.

2. B. Alexeev, M.A. Forbes, and J. Tsimerman, Tensor rank: Some lower and upper bounds, Computational Complexity (CCC), 2011 IEEE 26th Annual Conference on, June 2011, pp. 283-291.

3. Edoardo Ballico and Alessandro De Paris, Generic power sum decompositions and bounds for the Waring rank, arXiv:1312.3494 [math.AG], Dec 2013.

4. A. Białynicki-Birula and A. Schinzel, Representations of multivariate polynomials by sums of univariate polynomials in linear forms, Colloq. Math. 112 (2008), no. 2, 201-233.

5. Grigoriy Blekherman and Zach Teitler, On maximum, typical, and generic ranks, Math. Ann. 362 (2015), no. $3-4,1021-1031$

6. Weronika Buczyńska, Jarosław Buczyński, and Zach Teitler, Waring decompositions of monomials, J. Algebra 378 (2013), 45-57.

7. Jarosław Buczyński and Zach Teitler, Some examples of forms of high rank, Collectanea Mathematica (2015), DOI: $10.1007 / \mathrm{s} 13348-015-0152-0$

8. E. Carlini, M.V. Catalisano, L. Chiantini, A.V. Geramita, and Y. Woo, Symmetric tensors: rank, Strassen's conjecture and e-computability, arXiv:1506.03176 [math.AC], June 2015.

9. Enrico Carlini, Maria Virginia Catalisano, and Anthony V. Geramita, The solution to the Waring problem for monomials and the sum of coprime monomials, J. Algebra 370 (2012), 5-14.

10. Enrico Carlini, Cheng Guo, and Emanuele Ventura, Complex and real ranks of reducible cubics, arXiv: 1502.01051 [math.AC], February 2015.

11. Pierre Comon and Bernard Mourrain, Decomposition of quantics in sums of powers of linear forms, Signal Processing 53 (1996), no. 2-3, 93-107.

12. Alessandro De Paris, A proof that the maximal rank for plane quartics is seven, arXiv:1309.6475 [math.AG], Sep 2013.

13. Alessandro De Paris, Every ternary quintic is a sum of ten fifth powers, Internat. J. Algebra Comput. 25 (2015), no. 4, 607-631.

14. Anthony Iarrobino and Vassil Kanev, Power sums, Gorenstein algebras, and determinantal loci, Lecture Notes in Mathematics, vol. 1721, Springer-Verlag, Berlin, 1999, Appendix C by Iarrobino and Steven L. Kleiman. 
15. Joachim Jelisiejew, An upper bound for the Waring rank of a form, Arch. Math. (Basel) 102 (2014), no. 4, 329-336.

16. Johannes Kleppe, Representing a homogenous polynomial as a sum of powers of linear forms, Master's thesis, University of Oslo, 1999, http://folk.uio.no/johannkl/kleppe-master.pdf.

17. J. M. Landsberg, Tensors: Geometry and Applications, Graduate Studies in Mathematics, vol. 128, American Mathematical Society, Providence, RI, 2012.

18. , Geometric complexity theory: an introduction for geometers, Ann. Univ. Ferrara Sez. VII Sci. Mat. 61 (2015), no. 1, 65-117.

19. J.M. Landsberg and Zach Teitler, On the ranks and border ranks of symmetric tensors, Found. Comp. Math. 10 (2010), no. 3, 339-366.

20. Ran Raz, Tensor-rank and lower bounds for arithmetic formulas, J. ACM 60 (2013), no. 6, 40:1-40:15.

21. Bruce Reznick, On the length of binary forms, Quadratic and Higher Degree Forms (New York) (K. Alladi, M. Bhargava, D. Savitt, and P. Tiep, eds.), Developments in Math., vol. 31, Springer, 2013, pp. 207-232.

22. B. Segre, The Non-singular Cubic Surfaces, Oxford University Press, Oxford, 1942.

Erik Holmes, Department of Mathematics, University of Hawail at Manoa, 2565 McCarthy Mall (Keller Hall 401A), Honolulu, HaWait 96822, USA

E-mail address: eholmes@math.hawaii.edu

Paul Plummer, Department of Mathematics, University of Oklahoma, Norman, OK 730193103, USA

E-mail address: Paul.A.Plummer-1@ou.edu

Jeremy Siegert, Department of Mathematics, George Washington University, Monroe Hall, 2115 G St. NW, WAshington, DC 20052, USA

E-mail address: jsiegert@gwu.edu

Zach Teitler, Department of Mathematics, 1910 University Drive, Boise State University, BoISE, ID 83725-1555, USA

E-mail address: zteitler@boisestate.edu 